\title{
A Novel Emergency Vehicle Dispatching System
}

\author{
Chia-Ying Chen ${ }^{\dagger}$, Po-Yu Chen ${ }^{\ddagger}$, and Wen-Tsuen Chen ${ }^{\dagger \ddagger}$ \\ ${ }^{\dagger}$ Institute of Communications Engineering, National Tsing Hua University, Hsinchu 300, Taiwan \\ Institute of Information Science, Academia Sinica, Nankang, Taipei 115, Taiwan \\ Emails: joy90925@gmail.com, poyuchen@iis.sinica.edu.tw, chenwt@iis.sinica.edu.tw
}

\begin{abstract}
When accidents or disasters happen, emergency response time is always crucial. Most procedures in the existing emergency vehicle dispatching systems rely heavily on manual assignments. In this paper, we propose a novel emergency vehicle dispatching system to reduce emergency response time, which includes automatic emergency resource assignment and driving path planning to reduce unnecessary time delay caused by human misjudgment or the delay caused by traffic jam. As the traffic condition varies from time to time, the traffic information employed in the proposed method needs to trace back to several weeks prior to the dispatching time. Furthermore, we also propose a lane reservation scheme for emergency vehicles to minimize the crash risks. Before an emergency vehicle arrives at each road segment, Road Side Units start to disseminate the warning message at suitable time, so that other drivers could have enough time to take proper actions such as speed acceleration or switching lanes. Simulation results show that the proposed approach can largely reduce the traveling time of emergency vehicles comparing to the shortest path approach with sirens and lights to alert other drivers. With the traveling time as well as emergency resource assignment time being reduced, the emergency rescue teams would gain edges in rescuing lives and property.
\end{abstract}

Keywords-emergency dispatching system, lane reservation scheme, path planning, Vanets.

\section{INTRODUCTION}

In recent years, the problem of traffic congestion in urban area has become serious due to urbanization and tremendous increase in vehicles. This problem has largely contributed to more traffic accidents and caused longer emergency response time. For all emergency vehicles (EVs) such as police cars, fire engines, ambulances, it is important to arrive at the scene of an accident in time to save life and reduce damages. EVs have the highest priority on the road, and they might run over red lights or drive over speed under the pressure of time, and more unexpected accidents might be thus caused. Therefore, how to balance between safety and time urgency is an important issue in emergency management.

Currently, most EVs utilize sirens and lights to alert drivers of non-EV-Vehicles in the critical road segments ${ }^{1}$ (DNEVC) of their presence. Unfortunately, DNEVC often react too late or in a wrong way since the sound of sirens may be filtered between buildings, while the alerting lights of EVs could be blocked. In addition, even if the DNEVCs see the presence of EVs, they find it difficult to respond properly, in that those DNEVCs can never come up with a common thought and

\footnotetext{
${ }^{1}$ Critical road segments is the road segments which the EV is currently located in and is arriving at shortly.
}

drive coordinated. Especially, it is not easy for DNEVCs to give a pass way for EVs in rush hour.

After a field survey on many emergency handling agencies, including fire brigades, hospitals, and police stations, we discovered that the current EV dispatching systems rely heavily on personal judgments. Also, most current procedures such as hospital selection, choosing the path from fire brigade/hospital to accident scene and the path from accident scene to hospital, are operated mainly by on manual assignments, which may cause unnecessary time delay.

Many previous studies about path planning [1] [2] have been done with respect to common vehicles, which are essentially different from the major concerns in our study emergency vehicles - in the way that EVs have the first traffic priorities while common vehicles do not. In order to notify DNEVCs, previous works [3]-[5] adopted centralized or distributed schemes to disseminate warning messages. However, these authors did not discuss how to manage to form a suitable pass way for EV, as in how the DNEVCs can determine whether to switch to another lane or stay on the same lane even if they receive a warning message. Moreover, the authors consider neither the interaction between EVs nor complete emergency procedure. Two main reasons for driving time delay of EVs are emergency vehicle dispatching procedure and traffic congestion. Therefore, we design a comprehensive emergency dispatching system to reduce emergency response time considering various traffic conditions.

The emergency dispatching system proposed in this paper has the following main components: emergency resource assignment, path planning for EVs, and lane reservation for EVs. Simulation results show that the proposed system can reduce the emergency response time and the rescue teams have higher probability to rescue lives and property in time.

The remainder of this paper is organized as follows. In Section II we propose dispatching strategy and present some considerations about our proposed algorithms. Simulation results are provided in Section III. Finally, Conclusions and future works are given in Section IV.

\section{THE PROPOSED SYSTEM}

In this section, we introduce the proposed emergency vehicle dispatching system in detail, which comprises three major components: emergency resource assignment, path planning for EVs, and lane reservation for EVs. These three components are described in the following subsections.

The system architecture is shown in Fig. 1. In this system, we assume that there are sufficient Road Side Units (RSUs) 
placed on the roadside in the main roads and reaching to all road segments. Each vehicle is equipped with a Global Positioning System (GPS) device and an On Board Unit (OBU). RSUs collect road information (e.g. speed of vehicle, traffic flow) as well as broadcast warning messages. If some roads cannot be covered by RSUs, the system can broadcast warning message and collect road information through other means, such as smart phones via cellular networks and ITS (Intelligent Transportation System). The emergency system should have road conditions information, road traffic information, the emergency resource information (e.g. the number of patients each hospital can admit, the number of EVs each station can dispatch, the location of each EV on patrol). DNEVCs on the road get the location and time information of their own vehicles through their GPS devices, and receive warning messages from RSUs via OBUs. Since the EVs usually move on the main roads to escape congestion in narrow lanes, we remove small road segments from electronic map in our system in order to reduce processing time by decreasing insignificant information.

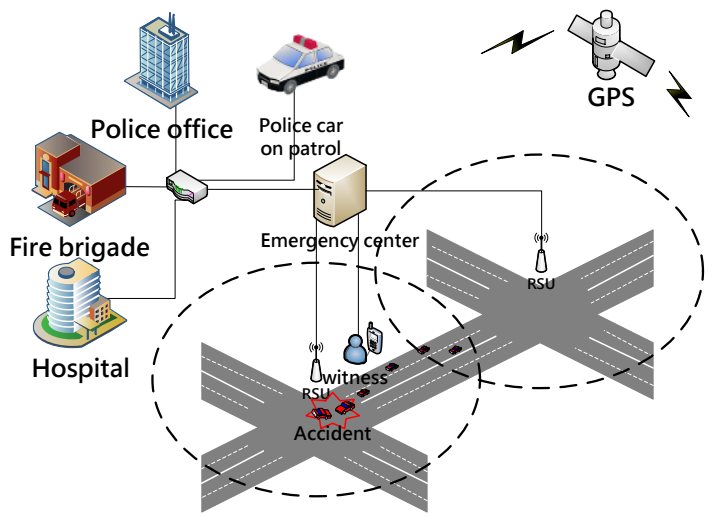

Fig. 1. System Architecture.

\section{A. Emergency resource assignment}

Many previous researches only focus on path planning for EVs, but the emergency resource assignment also plays an important role in the rescue process. In the real world, most of emergency dispatching procedures rely on personal judgments, and often this may cause unnecessary time delay. Sometimes the decisions by the staffs in emergency are not optimal, and misjudgements may cause more time delay. Moreover, if the emergency centre and a hospital do not coordinate in advance, the patient may need to transfer to another hospital after the ambulance arrives at the hospital. With the aforementioned reasons, we design an emergency vehicle dispatching system that includes the emergency resource assignment.

Upon receiving an event report, the staffs in emergency centre are only required to decide the number and types of EVs to be dispatched as well as confirm the location of the accident scene. Then, the system automatically looks for dispatching source of EVs in the area near accident scene. The searching range of dispatching sources is an area surrounding the accident scene as shown in Fig. 2. To accomplish the rescue mission, the emergency system executes the aforementioned process for each type of EVs in the area. The system first estimates the traveling time from each candidate dispatching source to the location of the accident. Then, it selects the one with the shortest traveling time. If the rescue mission needs more than one EV, the emergency system executes the process repetitively until sufficient number of $\mathrm{EVs}$ is found. Additionally, when the system cannot find enough EVs in Zone 1, it expands the searching range to Zone 2 and executes the process again. The responsibility block (RB) of each dispatching source (i.e. EV station in the example of Fig. 2) is defined as the area in which the authority designates the dispatching source taking the responsibility for accidents occurred. Zone 1 is defined as the area including the $\mathrm{RB}$ where the accident spot is located $\left(R B_{0}\right)$ together with those RBs encompassing $R B_{0}$, whereas Zone 2 consists of only those RBs immediately adjacent to the outer rim of Zone 1. In some rare cases where Zone 2 is not sufficient to fulfil the task, Zone 3 can be designated in a likewise manner.

The system estimates the traveling time based on the predicted traffic condition, and it concurrently plans the path from each candidate dispatching source to the accident scene. The traveling time estimation and path planning scheme will be described in next subsection. The emergency resource assignment can be summarized as follows:

Step 1. When the emergency system receives an emergency call, system staffs decide the number and the types of EVs that need to be dispatched.

Step 2. The system estimates the driving time from each dispatching source candidate to the accident scene in zone 1 and select the fastest one.

Step 3. If this rescue mission needs more than one EV, the system repeats step 2 again.

Step 4. If the system cannot find enough EVs in zone 1, the system expands its searching radius to zone 2 .

If there are patients need to be sent to a hospital, the system also chooses a suitable hospital that can admit the patient and the EV can reach in time.

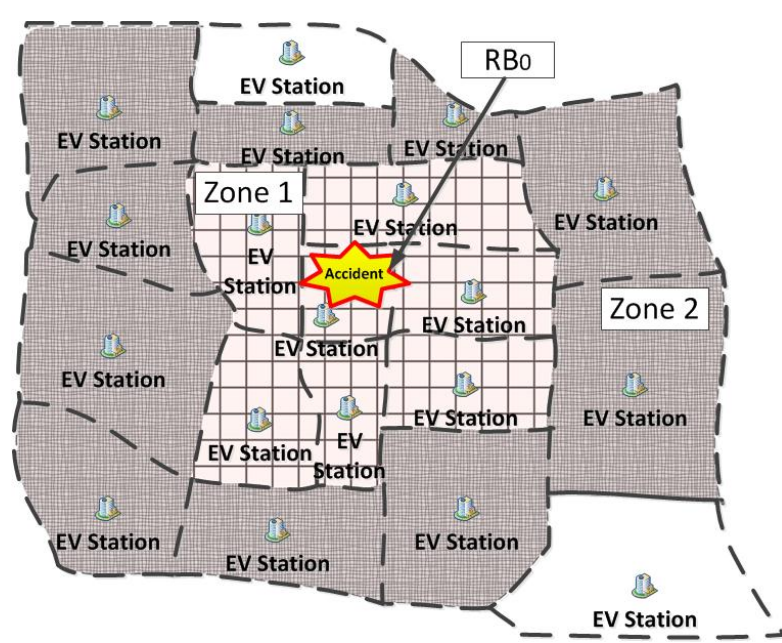

Fig. 2. The system computes the driving time from each dispatching source candidate to the accident scene within zones. 


\section{B. Path planning for emergency vehicles}

One of the main reasons for traveling time delay of EVs is traffic congestion which is affected by the distance between two places and the road traffic condition. When the shortest path is congested, drivers can arrive at destination by changing to other path with better traffic condition. Therefore, we adopt traveling time as the edge cost in our proposed path planning strategy based on Dijkstra's shortest path algorithm. In order to accurately estimate the traveling time of EVs on each road segment, we use current and historical traffic information gathered by the ITS for estimation.

We depict a road map as a directed graph $(V, E)$. An intersection and a road segment between two intersections are defined as a vertex and an edge of the graph respectively. For the purpose of finding the most suitable path for an EV, each edge is assigned with a cost equal to the traveling time of EV through the corresponding road segment, which is obtained with the estimated speed that can be calculated from the density of the road segment as will be further discussed in the following.

\section{Phase 1: Estimate the average speed of emergency vehicles}

Due to the fact that the traffic condition constantly changes, we have to consider both current and historical traffic information. The emergency system predicts the average speed of vehicles $V_{e s t_{x, t}}$ on road segment $x$ at time $t$ as follows:

$$
V_{e s t_{x, t}}=\alpha \times V_{x}+(1-\alpha) \times V_{h i s_{x, t}},
$$

where $V_{x}$ and $V_{\text {his }}$ are current and historical average speed of vehicles on road segment $x$ respectively. The former is directly updated from ITS and the latter reads from the historical data. The variable $\alpha$ is used to adjust the weight of current and historical information, and we run a simulation to find the most suitable value of $\alpha$. The symbol $t$ is the estimated time that EV arrives at road segment $x$.

After the estimated average speed of vehicles at time $t$ is obtained, the vehicle density $D_{x, t}$ of one lane of road segment $x$ at time $t$ can be estimated by the following equation:

$$
D_{x, t}=e^{b \times V_{e s t_{x, t}}}+C
$$

where $C$ and $b$ are parameters estimated by making a leastsquare regression to fit the historical data [11]. Based on the aforementioned equations, we can estimate the speed $V_{e m_{x, t}}$ of EVs at time $t$ according to the density information:

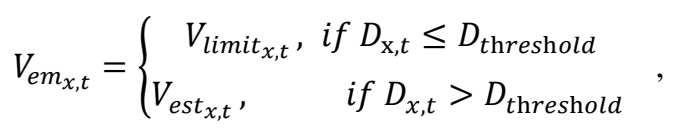

where $V_{\text {limit }_{x, t}}$ is the speed limit of road segment $x$, and $D_{\text {threshold }}$ denotes a density threshold. The authors in [12] analyzed the relationship between the lane-changing rate and traffic density. The lane-changing rate decreases as the traffic density increases. Based on the analysis, we set $D_{\text {threshold }}$ to
14 vehicles $/ \mathrm{km} / \mathrm{lane}$. When the density of a road segment is smaller than this threshold, the EVs can pass through the road segment at the speed limit.

After estimating the average speed of EVs on each road segment, we can obtain the required time of EVs to travel through road segment $x$ of length $L_{x}$ at time $t$ with the equation $T_{t}[x]=\frac{L_{x}}{V_{e m_{x, t}}}$, which will be used as the time cost of road segment $x$ for path planning in Phase 2 .

\section{Phase 2: Path planning}

The emergency system is a modified form of Dijkstra's shortest path algorithm to select the emergency path for an EV based on the traveling time cost of each road segment. When the EV travels through an intersection, the traveling time increases with $T_{\text {overhead }}$, which is the time overhead caused by traffic at intersection. The algorithm repeats until finding a path with the minimum traveling time and forms a series of intersections between the source node and the destination node.

\section{Lane reservation scheme}

After the path planning, the system obtains a path with a series of road segments. The system notifies DNEVCs in advance by sending warning messages through RSUs before the EV arrives at each road segment. Nevertheless, if the nearby RSUs emit notifications much earlier than the time critically needed, the circulation of the entire traffic system can be compromised. In contrast, if the RSUs fail to notify in time, the risk of failing the tasks of EVs soars. In the following, we provide road segment-based notification mechanism to estimate the alarm time of each RSU.

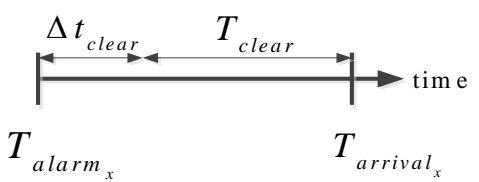

Fig. 3. The time line of lane reservation scheme.

The main idea is shown in Fig. 3. When a RSU begins to disseminate the warning message, DNEVCs take proper actions which must be finished before EVs arrival at this road segment. The estimated alarm time $T_{\text {alarm }_{x}}$ is:

$$
T_{\text {alarm }_{x}}=T_{\text {arrival }_{x}}-T_{\text {clear }_{x}}-\Delta t_{\text {clear }},
$$

where $T_{\operatorname{arrival}_{x}}$ is the expected arrival time of the EV on road segment $x ; T_{\text {clear }}$ and $\Delta t_{\text {clear }}$ is a system parameter of the reaction time of drivers [13], respectively. To find $T_{\text {alarm }}$, first we need calculation of $T_{\text {arrival }_{x}}$ and $T_{\text {clear }}$, which will be addressed separately in the following paragraphs.

As mentioned before, the expected arrival time will be earlier than the actual arrival time of EVs on each road segment in the planned path. To calculate the arrival time, we estimate the speed of an EV when it travels on different road segments. We assume that each road segment $S_{i}$ has three 
states: regular, clear, and ready. Each road segment is in regular state initially. The state of a road segment is in clear state when RSUs begin to notify DNEVCs that EVs are approaching. With the EVs entering on the road segment, the state of the road segment transits from clear state to ready state. After all EVs leave the road segment, the state returns to regular state. According to different states of each road segment $x$ in the planned path, the system expects the arrival time $T_{\text {arrival }_{x}}$ of an EV with the following equation:

$$
\begin{gathered}
T_{\text {arrival }_{x}}=\sum_{i=E V_{\text {pos }}}^{x-1} \frac{L_{i}}{V_{S_{i}}}, \\
V_{S_{i}}=\left\{\begin{array}{cc}
V_{\text {current }} & \text { if } S_{i}=\text { ready } \\
V_{\max } & \text { if } S_{i}=\text { clear } \\
\operatorname{Max}\left(V_{\text {avg }}, V_{\text {current }}\right) & \text { if } S_{i}=\text { regular }
\end{array} .\right.
\end{gathered}
$$

where $V_{\text {current }}$ is the current speed of the $\mathrm{EV}$, whereas $V_{\text {avg }}$ is the average speed of the EV in the planned path. $V_{\max }$ is the maximal speed of EVs predicted by the system in urban areas, while $E V_{\text {pos }}$ is the number of remaining road segments in the planned path that the EV travels on currently. The system calculates the arrival time periodically.

On receiving the warning message from RSUs, DNEVCs take proper actions. Reserving the innermost lane on road segment $x$ requires time $T_{\text {clear }}$ for DNEVCs, and it is estimated as follows:

$$
T_{\text {clear }_{x}}=\max \left(\frac{L_{x}}{V_{\text {est }}^{\prime}{ }_{x, t}}, \Delta t_{\text {clear }}\right),
$$

Since $L_{x}$ and $\Delta t_{\text {clear }}$ are pre-determined for road segment $x$, $T_{\text {clear }_{x}}$ can be determined by $V_{e s t_{x, t}}^{\prime}$, which is the estimated speed of the innermost lane. In responding to DNEVCs' switching lanes, the vehicle density $D_{x, t}$ changes; and subsequently the estimated speed of the innermost lane varies accordingly as follows:

$$
V_{e s t_{x, t}}^{\prime}=\frac{1}{b} \times \log \frac{D^{\prime} x, t}{C}, \text { where } D_{x, t}^{\prime}=\frac{N_{\text {est }} x_{, t}-N_{x, \text { change }}}{L_{x} \times \text { Lane }_{x}} .
$$

In equation $7, N_{x, \text { change }}$ denotes the number of DNEVCs who respond to the warning message by switching lanes. The system estimates $N_{x, \text { change }}$ as follows:

$$
N_{x, \text { change }}=i \times D_{x, t}^{2}+j \times D_{x, t}+k
$$

where $i, j$ and $k$ are empirical parameters estimated by fitting a quadratic regression line to the simulation result in [12] and listed in Table I.

After estimating the value of $T_{\text {arrival }_{x}}$ and $T_{\text {clear }}$, we can calculate the alarm time for each road segment and recalculate the alarm time whenever the state of road segments changes. Besides, RSUs near the departure location of the EV may not have enough time to broadcast warning messages in advance.
In this case, the value of $T_{\text {alarm }}$ is negative, and it entails that nearby RSUs should start to alarm immediately.

If someone is under life threatening or seriously injured, the system needs to plan a path for ambulance from the accident scene to the most proper hospital. Before the ambulance leaves the accident scene, RSUs broadcast the warning messages and notify DNEVCs in advance, so that the ambulance could travel in a safe and clear path at maximal possible speed to arrive at the hospital.

\section{SIMULATION RESULTS}

A traffic simulator written in $\mathrm{C}++$ is developed to evaluate our approach and compare to the shortest path approach in different traffic conditions. We simulate a $10 \mathrm{~km} \times 10 \mathrm{~km}$ area with $10 \times 10$ regular intersections and each road segment has two lanes. In this scenario, there are two hospitals, two fire brigades, two police stations, and two police cars on patrol in the area. We create accident scenes randomly and record the traveling time of the EV between the departure location and the destination. We assign different number of vehicles according to the congestion rate and use the congestion rate to express the ratio of the congested road segments in the area. A congested road segment means that its average speed is lower than $20 \mathrm{~km} / \mathrm{hr}$. In our simulation, we select congested road randomly. All simulation parameters are listed in Table I.

Table I : Simulation Parameters.

\begin{tabular}{|c|c|c|c|}
\hline Parameter & Value & Parameter & Value \\
\hline$\alpha$ & 0.8 & $V_{\text {max }}$ & $100 \mathrm{~km} / \mathrm{hr}$. \\
\hline $\mathrm{C}$ & 227 & $\Delta t_{\text {clear }}$ & $10 \mathrm{sec}$ \\
\hline $\mathrm{b}$ & -0.0581 & $i$ & -4.58 \\
\hline$T_{\text {overhead }}$ & $5 \mathrm{sec}$. & $j$ & 140 \\
\hline$D_{\text {threshold }}$ & 14 veh./km/lane & $k$ & -58.249 \\
\hline
\end{tabular}

We compare our real-time route guidance strategy with the shortest path approach, hybrid approach, and emergency path approach. The hybrid approach assumes an EV traveling on the shortest path with lane reservation scheme. The emergency path approach assumes that the EV travels on the shortest traveling time path with lane reservation scheme. Firstly, we compare the performance in both directions including the required time to arrive at accident scenes and the required time to return to the hospital.

We compare the average traveling time and speed of EVs with different approaches. The performance result shows that using the shortest path approach an EV needs to spend much more time than three other approaches. Due to page limitation, we show only the performance result for ambulance in Fig. 4. Fig. 4 (a) and Fig. 4 (c) depict the traveling time of an ambulance dispatched to accident scene and its traveling time from the accident scene to a hospital, respectively. Due to the fact that our approach avoids selecting congested road and choosing the most suitable dispatching source, it performs better than others. Both approaches with lane reservation scheme and estimation of traveling time of the ambulance can guarantee that the total traveling time is less than $30 \%$ of that 
of the shortest path approach even if the congestion rate is $70 \%$. Fig. 4 (b) and (d) shows the average speed of the EV for different congestion rates. The average speed of the EV in our approach is higher than those of three other approaches. Owing to the lane reservation scheme, the average speed in our approach is higher than $30 \mathrm{~km} / \mathrm{hr}$. even when the congestion rate exceeds $70 \%$.

In Fig. 4 (c) and (d), the simulation result shows that the proposed scheme performs as well as the emergency path approach. Since there are only two hospitals that can admit patients, the gain of the proposed method is limited to the choice of applicable hospitals.

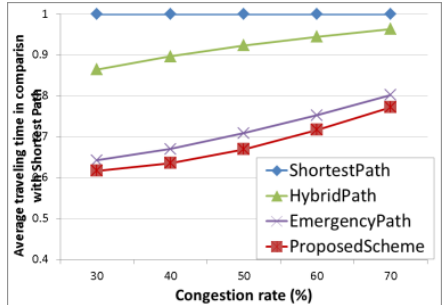

(a) Traveling time of an ambulance to the accident scene.

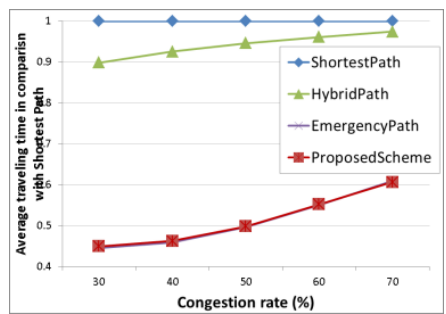

(c) Traveling time of an ambulance from accident scene to hospital.

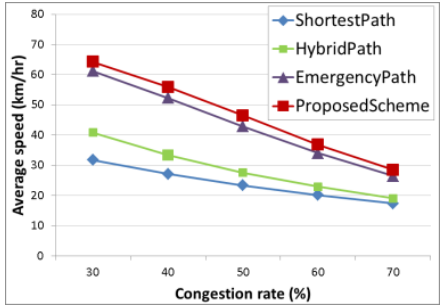

(b) Average speed of an ambulance to the accident scene.

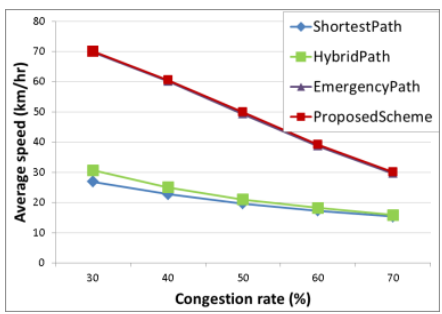

(d) Average speed of the ambulance travels from the accident scene to the hospital
Fig. 4. The comparison of average traveling time and speed of EVs.

\section{CONCLUSIONS}

In this paper, we propose a real-time route guidance strategy to reduce the traveling time of EVs. Our strategy consists of emergency resource assignment, path planning and reverse path planning for EVs. Since the traffic condition constantly changes, we use current and historical traffic information to plan a path. Furthermore, we propose a lane reservation scheme for EVs. By preserving the lane on the road in time with the warning messages disseminated to the planned path, the EVs get edges in accomplishing the tasks.

Future studies on the present issue can include considerations of more severe scenarios, such as disasters where a large number of EVs are required. Amendments to the current dispatching strategy could be in need for those scenarios. The ultimate goal of the current study is to put the proposed dispatching system into practical uses. The proposed dispatching system requires further advancements in social infrastructure. More efforts need to be put on real-world considerations.

\section{REFERENCES}

[1] E. W. Dijkstra, "A Note on Two Problems in Connection with Graphs," Numeriche Mathematik, vol. 1, 1959.

[2] E. J. Schmitt and H. Jula, "Vehicle route guidance systems: Classification and comparison," in Proceedings of IEEE Intelligent Transportation Systems Conference, Sep. 2006.

[3] J. Woodbridge, M. Lan, G. Pau, M. Gerla, and M. Sarrafzadeh, "Hero: Hybrid emergency route-opening protocol," in Proceedings of the $8^{\text {th }}$ IEEE International Conference on Pervasive Computing and Communications Workshops (PerComW07), March 2010.

[4] C.-M. Huang, C.-C. Yang, C.-Y. Tseng and C.-H. Chou, "A centralized traffic control mechanism for evacuation of emergency vehicles using the DSRC protocol," in Proceedings of the International Symposium on Wireless Pervasive Computing, Feb. 2009.

[5] S. Rizvi, S. Olariu, M. Weigle, and M. Rizvi, "A novel approach to reduce traffic chaos in emergency and evacuation scenarios," in Proceedings of the IEEE 66th Vehicular Technology Conference (VTC-2007 Fall), Oct. 2007.

[6] R. S'anchez-Mangas, A. Garc'1a-Ferrrer, A. de Juan, and A. M. Arroyo, "The probability of death in road traffic accidents. How important is a quick medical response?” Accident Analysis \& Prevention, vol. 42, no. 4, 2010.

[7] R. P. Gonzalez, G. R. Cummings, H. A. Phelan, M. S. Mulekar, and C. B. Rodning, "Does increase emergency medical services prehospital time affect patient mortality in rural motor vehicle crashes? A statewide analysis," The American Journal of Surgery, vol. 197, no. 14, 2009.

[8] B. Gong, Z. Yang, and C. Lin, "Dispatching optimization and routing guidance for emergency vehicles in disaster," in Proceedings of the IEEE International Conference on Automation and Logistics, Aug. 2009.

[9] A. Jotshi, Q. Gong, and R. Batta, "Dispatching and routing of emergency vehicles in disaster mitigation using data fusion," in SocioEconomic Planning Sciences, vol. 43, no. 1, 2009.

[10] R. C. Vlad, C. Morel, J. Y. Morel, and S. Vlad, "A learning real-time routing system for emergency vehicles," in Proceedings of the IEEE International Conference on Automation, Quality and Testing, Robotics, vol. 3, May 2008.

[11] S. L. Dhingral and I. Gull, "Traffic flow theory historical research perspectives," in Proceedings of Green shields Symposium, July 2008.

[12] A. Kesting, M. Treiber, and D. Helbing, "General Lane-Changing Model MOBIL for Car-Following Models," Transportation Research Record, vol. 1999, March 2007.

[13] D. Salvucci and A. Liu, "The Time Course of a Lane Change: Driver Control and Eye Movement Behavior." Transportation Research, Part F, 2002, vol. 5, no. 2. 